\title{
PARAMETRIC IDENTIFICATION OF REACTION WHEEL PENDULUMS WITH ADAPTIVE CONTROL
}

\author{
Aleksandr Alyshev \\ Control Systems and Robotics \\ ITMO University \\ Russia \\ aalyshev@yandex.ru
}

\author{
Natalia Dudarenko \\ Control Systems and Robotics \\ ITMO University \\ Russia \\ dudarenko@yandex.ru
}

\author{
Vitaly Melnikov \\ ITMO University \\ Mining University \\ Russia \\ v.g.melnikov@yandex.ru
}

Article history:

Received 18.06.2018, Accepted 03.09.2018

\begin{abstract}
The paper proposes a new adaptive method for identification of the axial moment of inertia for a two degrees of freedom reaction wheel pendulum with viscous friction in suspension bearings. The pendulum contains a rod, a controlled DC motor and a flywheel attached to the rotor shaft. One end of the rod rotates in hinge bearing. The controlled DC motor with a flywheel is attached to the other end of the rod. The angles of rotation of the rod and of the controlled flywheel are measured by encoders. The proposed adaptive method is based on an energy algorithm with reversible symmetric motions of the pendulum system. The symmetric motions are used to eliminate the influence of dissipative factors on the results of identification. The results of computer modelling and experimental results show high accuracy of the proposed identification method.
\end{abstract}

\section{Key words}

Moment of inertia identification, reaction wheel pendulum, consecutive compensator, adaptive control.

\section{Introduction}

The problem of precise identification of moments of inertia (MOI) of solid systems operating under conditions of unknown friction and environmental resistance is an actual problem of mechanics and control theory. Identification of inertial parameters of the various constructions or of their complicated components is especially important in robotics. In some cases it is possible to estimate inertia parameters theoretically by using CAD programs, but in practice the thorough model of the system is often unknown, or requires a time-consuming process to obtain it.
The inertial parameters can be estimated experimentally [Tikhonov and Tkhai, 2016, Ovchinnikov et al., 2017, Andrievskiy and Boikov, 2017] using various time and frequency domain methods [Almeida et al., 2007, Ivanov and Melnikov, 2015]. In the time domain methods including the pendulum method or its various modifications [Pandit et al., 1992], the inertial properties of a weighted body are determined relative to the period of its oscillations. Despite the fact that these methods are widely used, they are not accurate in the case of significant friction in the system or significant resistance of the environment. Frequency domain methods (IRM and DPPIM) and modal methods [Almeida et al., 2008] are based on measuring the motion of a solid body in bi-filar or multi-filar suspensions, Stewart platforms, etc. [Malekjafarian et al., 2016, Barreto and Muñoz, 2010, Hou et al., 2009]. These methods also have drawbacks. For example, multi-filar suspensions create a significant measurement error due to additional unwanted movements in different directions, require accurate calibration and additional time for preparation of the test. In control theory, inertial parameters are estimated using various identification methods based on the analysis of the motion [Gobbi et al., 2011, Garćra-Alarćon et al., 2012].

In this article, we present a new adaptive method for identifying inertial parameters of solids on special symmetrical program motions. The method uses an energy approach [Fradkov and Andrievsky, 2006,Fradkov and Andrievsky, 2004] and is based on an energy algorithm [Alyshev et al., 2015,Melnikov, 2012,Dudarenko et al., 2014]. This algorithm uses a two-step reversible symmetric motion, containing two motions with approximately equal energy dissipation. We use robust adaptive control with the high-gain feedback principle to provide the desired program motions. Using this method, we do not need a friction evaluation to accurately identify the MOI. 
The paper is organized as follows. In Section 2 we describe the construction of the pendulum. In Section 3 we obtain a general equation for calculating the MOI of the rod. In Section 4 we present an algorithm for controlling the program symmetrical motions of the pendulum and give the results of mathematical modelling and identification of MOI of the rod in MATLAB. After that we compare the obtained results with the results of existing methods. In Section 5 we present the results obtained at our experimental device. In Section 6 we conclude our paper with concluding remarks.

\section{Problem Formulation}

We will present and demonstrate our method on a system with two degrees of freedom (DOF) called the reaction-wheel pendulum [Block et al., 2007, Spong et al., 2001].
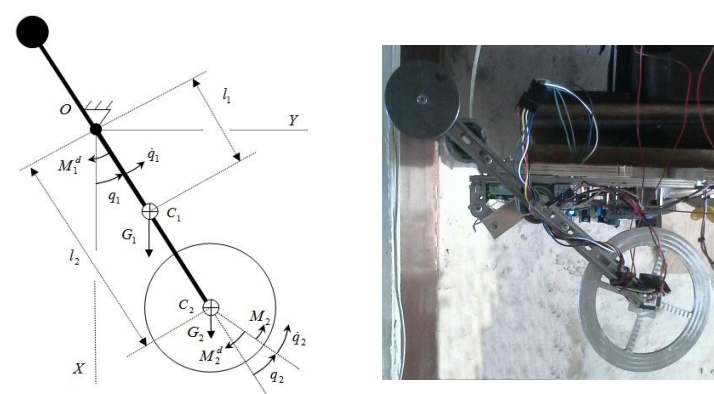

Figure 1. Reaction Wheel Pendulum

The system consists of two kinematic groups of elements. The first group consists of a rod with the stator of the motor and the housing of the motor gearbox with the central MOIs that are denoted by $J_{\text {rod }}, J_{s}$ and $J_{g h}$. The first group performs a rotation motion around the fixed horizontal axis $O$ in the plane $O X Y$. The second group consists of a rotor of the motor and a flywheel (a homogeneous disk) with the central MOIs that are denoted by $J_{r}$ and $J_{2}$. The second group performs a plane motion. Note that we will use the second group as a measuring device to identify the element of the first group, so the parameters of the second group should be known.

The device is shown in Fig. 1, where $q_{1}$ is the absolute angle of rotation of the rod; $q_{2}$ is the relative angle of rotation of the flywheel; $\dot{q}_{1}$ is the angular velocity of the rod; $\dot{q}_{2}$ is the angular velocity of the flywheel. We assume that the position $l_{1}$ of the center of gravity (COG) of the rod $C_{1}$ and the position $l_{2}$ of the COG of the second element $C_{2}$ and of the motor $C_{2}^{\prime}$ are known. We denote the torque of viscous friction in the suspension bearing by $M_{1}^{d}$; the electromagnetic torque of the motor by $M_{2}$; the gravity forces of the elements by $G_{1}$, $G_{2}$ and of the motor $G_{2}^{\prime}$.
We will identify the MOI $J_{1}=J_{\text {rod }}+J_{g h}+J_{s}+$ $m_{1} l_{1}^{2}+\left(m_{2}^{\prime}+m_{2}\right) l_{2}^{2}$ and dissipative parameter using symmetric program motion and then we will proof the MOI identification accuracy by adding the additional body with known moment of inertia $J_{e}=J_{b o d y}+$ $m_{\text {body }} l_{2}^{2}$. The program motion consists of a slowed part and a symmetrical inverse accelerated part. Both parts are performed in the same angular interval $q_{11} \leq$ $q_{1} \leq q_{12}$ after the preliminary accelerated motion. We use the high-resolution sensors to measure the angle of rotation of the flywheel and the pendulum. The program motion is performed under conditions of substantial viscous friction in bearings.

\section{Energy Algorithm in MOI Identification Prob- lem}

Here we introduce the MOI identification procedure for a system with a measuring drive with a flywheel. The work of inertial torques changes its sign when the pendulum passes into reverse motion; the work of dissipative torques is always negative. This leads to the possibility of an analytical purification of MOI identification procedure from dissipative torques. Using the principle of the energy theorem for two motions, we get

$$
\begin{gathered}
\left(T_{12}+\Pi_{12}\right)-\left(T_{11}+\Pi_{11}\right)=A_{1}+B_{1}+D_{1} \\
\left(T_{22}+\Pi_{22}\right)-\left(T_{21}+\Pi_{21}\right)=A_{2}+B_{2}+D_{2},
\end{gathered}
$$

here $T_{11}, T_{12}, T_{21}, T_{22}$ and $\Pi_{11}, \Pi_{12}, \Pi_{21}, \Pi_{22}$ are the nodal values of the kinetic and potential energies of the pendulum system; $A_{1}$ and $A_{2}$ are the work of the motor torque in the considered angular interval on the forward $\left[q_{11}, q_{12}\right]$ and on the inverse $\left[q_{13}, q_{14}\right]$ motions; $B_{1}$ and $B_{2}$ are negative work of unknown torques of viscous friction in the bearing $O$ on two program motions; $D_{1}$ and $D_{2}$ are negative work of known torques of viscous friction in the bearings of the measuring motor. Note that the potential energy of the system at the same angular positions has the same values, i.e. $\Pi_{11}=\Pi_{22} \equiv \Pi_{1}$ and $\Pi_{12}=\Pi_{21} \equiv \Pi_{2}$.

We assume that the work of dissipative torques in bearings on symmetrical pendulum motions are approximately the same, i.e. $B_{1} \approx B_{2}<0$. Subtracting (2) from (1), we obtain an equation that does not contain dissipative torques:

$$
\begin{aligned}
& T_{12}-T_{11}+T_{21}-T_{22}= \\
& \quad=2 \Pi_{1}-2 \Pi_{2}+A_{1}-A_{2}+D_{1}-D_{2} .
\end{aligned}
$$

The equation (3) contains the nodal values of the kinetic and potential energies and consumption of elec- 
trical energy at the angular interval $\left[q_{12}, q_{11}\right]$ :

$$
\begin{aligned}
& 2 T_{11}=a_{11} \dot{q}_{11}^{2}+2 a_{12} \dot{q}_{11} \dot{q}_{21}+a_{22} \dot{q}_{21}^{2}, \\
& 2 T_{12}=a_{11} \dot{q}_{12}^{2}+2 a_{12} \dot{q}_{12} \dot{q}_{22}+a_{22} \dot{q}_{22}^{2}, \\
& 2 T_{21}=a_{11} \dot{q}_{13}^{2}+2 a_{12} \dot{q}_{13} \dot{q}_{23}+a_{22} \dot{q}_{23}^{2}, \\
& 2 T_{22}=a_{11} \dot{q}_{14}^{2}+2 a_{12} \dot{q}_{14} \dot{q}_{24}+a_{22} \dot{q}_{24}^{2}, \\
& a_{11}=J_{1}+J_{2}+J_{r} ; a_{12}=J_{r} i^{-1}+J_{2} \\
& a_{22}=J_{r} i^{-2}+J_{2},
\end{aligned}
$$

$$
\begin{aligned}
& \Pi_{1}=b\left(1-\cos q_{11}\right) \\
& \Pi_{2}=b\left(1-\cos q_{12}\right),
\end{aligned}
$$

where $m_{1}, m_{2}^{\prime}$ and $m_{2}$ are the masses of the rod, the motor and second elements, $g$ is the gravitational acceleration; $\dot{q}_{21}, \dot{q}_{22}$ and $\dot{q}_{23}, \dot{q}_{24}$ are the angular velocities of the flywheel at the beginning and at the end of two symmetrical movements.

Substituting (4) for (3) and collecting the terms, we obtain the equation for MOI $\hat{J}_{1}$ :

$$
\begin{gathered}
\quad \hat{J}_{1}=\left(2\left(A_{1}-A_{2}\right)+2\left(D_{1}-D_{2}\right)+4 b\left(\cos q_{12}-\cos q_{11}\right)-\right. \\
-a_{22}\left(\dot{q}_{22}^{2}+\dot{q}_{23}^{2}-\dot{q}_{21}^{2}-\dot{q}_{24}^{2}\right)-2 a_{12}\left(\dot{q}_{12} \dot{q}_{22}+\dot{q}_{13} \dot{q}_{23}-\dot{q}_{11} \dot{q}_{21}-\right. \\
\left.\left.-\dot{q}_{14} \dot{q}_{24}\right)\right) /\left(\dot{q}_{12}^{2}+\dot{q}_{13}^{2}-\dot{q}_{11}^{2}-\dot{q}_{14}^{2}\right)-J_{2}-J_{r}-m_{2} l_{2}^{2},
\end{gathered}
$$

where $b=g\left(m_{1} l_{1}+\left(m_{2}^{\prime}+m_{2}\right) l_{2}\right)$; the works $A_{1}, A_{2}, D_{1}, D_{2}$ on the time interval $t \in\left[t_{10}, t_{11}\right]$ of the direct motion and on the time interval $t \in\left[t_{20}, t_{21}\right]$ of the inverse motion are defined by $A_{j}=\int_{t_{j 0}}^{t_{j 1}} M(t) i^{-1} \dot{q}_{2}(t) d t, D_{j}=$ $-\int_{t_{j 0}}^{t_{j 1}} M_{2}^{d}(t) i^{-1} \dot{q}_{2}(t) d t, \quad j=1,2$ or, we can calculate the work $A_{1}, A_{2}, D_{1}, D_{2}$ from the very beginning of the symmetric motion using $A_{j}=$ $i^{-1}\left(\int_{0}^{t_{j 1}} M(t) \dot{q}_{2}(t) d t-\int_{0}^{t_{j 0}} M(t) \dot{q}_{2}(t) d t\right)$.

Suppose that the friction torque $M_{1}^{d}$ acting in the bearing $O$ has an unknown significant viscous friction coefficients $f_{1}: M_{1}^{d}=f_{1} i^{-1} \dot{q}_{1}$. So, the works $B_{1}$ and $B_{2}$ are defined by: $B_{i}=-\int_{t_{i 0}}^{t_{i 1}} M_{1}^{d}(t) \dot{q}_{1}(t) d t, \quad i=1,2$. Summarize (2) and (1), supposing that the inertial parameter is eliminated from the calculation, we obtain an equation for the coefficients $f_{1}$ :

$$
\begin{gathered}
\hat{f}_{1}=\left(2\left(A_{1}+A_{2}+D_{1}+D_{2}\right)-2 a_{12}\left(\dot{q}_{12} \dot{q}_{22}-\right.\right. \\
\left.-\dot{q}_{11} \dot{q}_{21}+\dot{q}_{14} \dot{q}_{24}-\dot{q}_{13} \dot{q}_{23}\right)-a_{22}\left(\dot{q}_{22}^{2}-\dot{q}_{21}^{2}+\right. \\
\left.\left.+\dot{q}_{24}^{2}-\dot{q}_{23}^{2}\right)\right) /\left(2 i^{-1}\left(\int_{t_{10}}^{t_{11}} \dot{q}_{1}^{2}(t) d t+\int_{t_{20}}^{t_{21}} \dot{q}_{1}^{2}(t) d t\right)\right) .
\end{gathered}
$$

\section{Example}

The example demonstrates a proposed adaptive energy method for MOI identification.

\subsection{Mathematical Model of the Pendulum}

Using the Lagrange method, we obtain a mathematical model of a pendulum with two DOFs in the form of a system of two differential equations with constant parameters

$$
\begin{aligned}
& a_{11} \ddot{q}_{1}+a_{12} \ddot{q}_{2}=-b \sin q_{1}-M_{1}^{d} \\
& i a_{12} \ddot{q}_{1}+i a_{22} \ddot{q}_{2}=M-M_{2}^{d} .
\end{aligned}
$$

Let the torque of the motor $M_{2}$ be an approximately linear function of the angular velocity of the rotor: $M_{2}=c_{1} u-c_{2} \dot{q}_{2} ; M$ is the torque with deadzone of the motor; $i^{-1}$ is the transmission gear ratio; $M_{2}^{d}=$ $f_{2} i^{-1} \dot{q}_{2}+f_{3} \operatorname{sign}\left(i^{-1} \dot{q}_{2}\right)$ is the motor friction. The DC motor has the deadzone

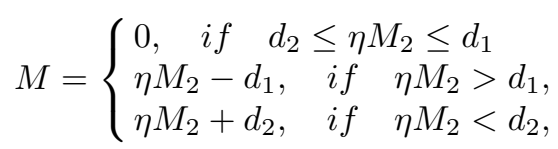

where $\eta$ is the electrical efficiency of the motor.

We assume that the coefficients $f_{2}$ and $f_{3}$ of viscous and Coulomb friction in the motor bearings with the gearbox; deadzone parameters $d_{1}, d_{2}$ and the coefficients $\eta, c_{1}$ and $c_{2}$ are known from the preliminary identification of the motor mathematic model.

We use the control signal $u_{1}=\left(c_{1} \eta\right)^{-1} f_{3} \operatorname{sign}\left(i^{-1} \dot{q}_{2}\right)$ to neutralize Coulomb friction in the bearing $O$ and than we get

$$
\begin{array}{r}
\ddot{q}_{1}=\left(a_{22} a_{11}-a_{12}^{2}\right)^{-1}\left(-a_{22}\left(b \sin q_{1}-M_{1}^{d}\right)-\right. \\
\left.-a_{12} i^{-1}\left(\eta M_{2}-f_{2} i^{-1} \dot{q}_{2}\right)\right), \\
\ddot{q}_{2}=\left(a_{22} a_{11}-a_{12}^{2}\right)^{-1}\left(a_{11} i^{-1}\left(\eta M_{2}-f_{2} i^{-1} \dot{q}_{2}\right)+\right. \\
\left.+a_{12}\left(b \sin q_{1}+M_{1}^{d}\right)\right),
\end{array}
$$

or in the Cauchy form:

$$
\begin{aligned}
& \dot{x}_{1}=x_{2}, \\
& \dot{x}_{2}=-\alpha_{3} x_{2}-\alpha_{2} \sin x_{1}+\alpha_{7} x_{4}-\alpha_{4} u_{2}, \\
& \dot{x}_{3}=x_{4}, \\
& \dot{x}_{4}=\alpha_{8} x_{2}+\alpha_{6} \sin x_{1}-\alpha_{9} x_{4}+\alpha_{5} u_{2},
\end{aligned}
$$


where

$$
\begin{aligned}
& x_{1}=q_{1}, \quad x_{2}=\dot{q}_{1}, \quad x_{3}=q_{2}, \quad x_{4}=\dot{q}_{2}, \\
& u_{0}=u_{1}+u_{2}+u_{3}, \alpha_{1}=\left(a_{22} a_{11}-a_{12}^{2}\right)^{-1}, \\
& \alpha_{2}=\alpha_{1} a_{22} b, \alpha_{3}=\alpha_{1} a_{22} f_{1} i^{-1}, \\
& \alpha_{4}=\alpha_{1} a_{12} i^{-1} \eta c_{1}, \\
& \alpha_{5}=\alpha_{4} a_{11} a_{12}^{-1}, \\
& \alpha_{6}=\alpha_{2} a_{12} a_{22}^{-1}, \\
& \alpha_{7}=\alpha_{1} a_{12} i^{-1}\left(c_{2} \eta+f_{2} i^{-1}\right), \\
& \alpha_{8}=\alpha_{3} a_{12} a_{22}^{-1}, \alpha_{9}=\alpha_{7} a_{11} a_{12}^{-1},
\end{aligned}
$$

here $\alpha_{1} \ldots \alpha_{9}$ are positive coefficients; $x_{1}$ is the output of the system; $u_{3}$ is the DC-motor deadzone compensation signal defined as:

$$
u_{3}=\left\{\begin{array}{l}
d_{1}\left(c_{1} \eta\right)^{-1}, \quad \text { if } u_{1}+u_{2}>0 \\
0, \quad \text { if } u_{1}+u_{2}=0 \\
d_{2}\left(c_{1} \eta\right)^{-1}, \quad \text { if } u_{1}+u_{2}<0
\end{array}\right.
$$

The initial deviation from the equilibrium position of the pendulum can be set manually, or by using an additional device or by using preliminary controlled motions [Andrievskiy, 2004, Andrievskiy, 2011, Bobtsov et al., 2011, Fradkov et al., 2012, Tikhonov, 2000]. The compact form of the equations of motion with respect to dimensionless time is presented in [Beznos et al., 2003a, Beznos et al., 2003b].

Here we develop a control for nonlinear system (10). The control should ensure the symmetry of the program motion $y(t)=x_{1}(t)$ of the pendulum for the limited reference signal $y_{m}(t)$ as $\lim _{t \rightarrow \infty}\left|y(t)-y_{m}(t)\right|<$ $\delta$. We use the algorithm proposed in [Bobtsov et al., 2011, Bobtsov and Pyrkin, 2008], where only the output variable is measured. The non-linear system (10) in the input-output representation has the form

$$
y=\frac{r(p)}{a(p)} u_{2}+\frac{h(p)}{a(p)} \varphi(y)
$$

where $r(p)=r_{m} p^{m}+r_{m-1} p^{m-1}+\ldots+r_{1} p+r_{0}$, $r_{m}>0, a(p)=p^{n}+a_{n-1} p^{n-1}+\ldots+a_{1} p+a_{0}$, $h(p)=p^{n}+h_{n-1} p^{n-1}+\ldots+h_{1} p+h_{0}$ are polynomials with unknown parameters; $m \leq n-1 ; \rho=n-m$ is the maximum relative degree of transfer function $\frac{r(p)}{a(p)}$. The polynomial $r(p)$ in (13) is Hurwitz and $\varphi(y)$ is the unknown function that satisfies the assumptions $\varphi(0)=0,-C_{0} \leq \frac{\varphi(y)}{y} \leq C$ for any $y \neq 0$. The equation for the tracking error $e(t)$ has the form

$$
e(t)=y_{m}(t)-x_{1}(t)
$$

We define the control $u(t)$ in the form

$$
u(t)=-(\nu+\kappa) \alpha(p) \xi_{1}(t), \quad \nu>\nu_{0}
$$

$$
\left\{\begin{array}{l}
\dot{\xi}_{1}(t)=\sigma \xi_{2}(t), \\
\dot{\xi}_{2}(t)=\sigma \xi_{3}(t), \\
\ldots \\
\ddot{\xi}_{\rho-1}(t)=\sigma\left(k_{1} e(t)-\right. \\
\left.-k_{1} \xi_{1}(t)-\ldots-k_{\rho-1} \xi_{\rho-1}(t)\right),
\end{array}\right.
$$

where the polynomial $\alpha(p)$ of order $\rho-1$ is chosen so that the polynomial $a(p)+\nu r(p) \alpha(p)$ is Hurwitz. The positive parameter $\kappa$ is determined from the conditions for neutralizing the nonlinearity $\varphi(y)$. The coefficients $\sigma>\nu+\kappa, k_{i}$ are determined from the asymptotic stability conditions for the system (16).

Now we define the coefficients $\nu, \kappa, \sigma$ of the controller. The parameter $\tilde{k}=\nu+\kappa$ can be tuned as follows:

$$
\tilde{k}(t)=\int_{t_{0}}^{t} \lambda(\tau) d \tau
$$

where the function $\lambda(t)$ is defined as follows

$$
\lambda(t)=\left\{\begin{array}{ll}
\lambda_{0}, & \left|e(t) W_{f}(p)\right|>\delta_{0} \\
0, & \left|e(t) W_{f}(p)\right| \leq \delta_{0}
\end{array} ; \quad \lambda_{0}>0 .\right.
$$

The parameter $\sigma$ is calculated using the following algorithm

$$
\sigma(t)=\sigma_{0} \tilde{k}^{2}(t), \sigma_{0}>0
$$

Since the system (13), where $h(p)=-\alpha_{2} p+\alpha_{6} \alpha_{7}-$ $\alpha_{2} \alpha_{9}, a(p)=p^{3}+\left(\alpha_{9}+\alpha_{3}\right) p^{2}+\left(\alpha_{3} \alpha_{9}-\alpha_{7} \alpha_{8}\right) p$, $r(p)=-\alpha_{4} p-\alpha_{4} \alpha_{9}+\alpha_{5} \alpha_{7}, \varphi(y)=\sin y$ has the maximum relative degree $\rho=2$, then the controller (15) - (16) with $\alpha=p+1, k_{1}=1$ has the form

$$
\begin{aligned}
& u(t)=-(p+1) \tilde{k}(t) \xi_{1}(t)= \\
&=-\left(\dot{\tilde{k}}(t) \xi_{1}(t)+\tilde{k}(t) \dot{\xi}_{1}(t)+\tilde{k}(t) \xi_{1}(t)\right) \\
& \dot{\xi}_{1}(t)=\sigma\left(-\xi_{1}(t)+e(t)\right)
\end{aligned}
$$

\subsection{Alternative Method}

Another approach is to estimate MOI using the inverse controller (adaptive backstop control) proposed in [Krstic et al., 1995, Benaskeur and Desbiens, 1998, Chiu et al., 2011, Ebrahim and Murphy, 2005] with stability estimation by Lyapunov function [Alyshev et al., 2018,Melnikov, 2010]. Let $e_{1}$ be the tracking error (14) and $e_{2}$ be the second error defined as

$$
e_{2}=\hat{x}_{2}-\beta_{1}-\dot{y}_{m},
$$


where $t$ is omitted for brevity. Then the derivatives of the tracking errors $e_{1}$ and $e_{2}$ have the form

$$
\dot{e}_{1}=\hat{\dot{x}}_{1}-\dot{y}_{m}, \quad \dot{e}_{2}=\hat{\dot{x}}_{2}+n_{1} \dot{e}_{1}-\ddot{y}_{m}
$$

where $\beta_{1}=-n_{1} e_{1}$ is the virtual control signal.

We choose the first Lyapunov function as follows

$$
V_{1}=\frac{1}{2} e_{1}^{2}
$$

Taking the derivative of the Lyapunov function $V_{1}$, we obtain

$$
\begin{aligned}
& \dot{V}_{1}=e_{1} \dot{e}_{1}=e_{1}\left(\hat{\dot{x}}_{1}-\dot{y}_{m}\right)= \\
& \quad=e_{1}\left(\hat{x}_{2}-\dot{y}_{m}\right)=e_{1}\left(e_{2}+\beta_{1}\right)=e_{1} e_{2}-n_{1} e_{1}^{2}
\end{aligned}
$$

If $e_{2}=0$, then we get $\dot{V}_{1}=-n_{1} e_{1}^{2} \leq 0$ where $n_{1}>0$ and $\hat{\dot{x}}_{1}=\hat{x}_{2}$.

We choose a new candidate for the Lyapunov function $V_{2}$ as

$$
V_{2}=V_{1}+\frac{1}{2} e_{2}^{2}+\frac{1}{2 \gamma_{1}} e_{\alpha_{3}}^{2}+\frac{1}{2 \gamma_{2}} e_{\alpha_{2}}^{2}
$$

Taking the derivative of the function, we get

$$
\begin{aligned}
& \dot{V}_{2}=\dot{V}_{1}+e_{2} \dot{e}_{2}+\frac{1}{\gamma_{1}} e_{\alpha_{3}} \dot{e}_{\alpha_{3}}+\frac{1}{\gamma_{2}} e_{\alpha_{2}} \dot{e}_{\alpha_{2}}= \\
& =-n_{1} e_{1}^{2}+e_{2}\left(e_{1}+x_{2}+n_{1} \dot{e}_{1}-\ddot{y}_{m}\right)-\frac{1}{\gamma_{1}} e_{\alpha_{3}} \alpha_{3}- \\
& -\frac{1}{\gamma_{2}} e_{\alpha_{2}} \dot{\hat{\alpha}} \alpha_{2}=-n_{1} e_{1}^{2}+e_{2}\left(e_{1}-\alpha_{3} \hat{x}_{2}-\alpha_{2} \sin x_{1}+\right. \\
& \left.+\alpha_{7} x_{4}-\alpha_{4} u_{2}+n_{1} \dot{e}_{1}-\ddot{y}_{m}\right)+e_{\alpha_{3}}\left(\frac{-\hat{\dot{\alpha}}_{3}}{\gamma_{1}}-e_{2} \hat{x}_{2}\right)+ \\
& +e_{\alpha_{2}}\left(\frac{\hat{-\hat{\alpha}} \alpha_{2}}{\gamma_{2}}-e_{2} \sin x_{1}\right),
\end{aligned}
$$

where $e_{\alpha_{3}}=\alpha_{3}-\hat{\alpha}_{3}, e_{\alpha_{2}}=\alpha_{2}-\hat{\alpha}_{2}$ are the parameter estimations errors; $\dot{e}_{\alpha_{3}}=-\dot{\hat{\alpha}}_{3}, \dot{e}_{\alpha_{2}}=-\dot{\hat{\alpha}}_{2}$ are the derivatives of parameter estimation errors.

We apply the control signal $u$ in the following form

$u=\hat{\alpha}_{4}^{-1}\left(e_{1}-\hat{\alpha}_{3} \hat{x}_{2}-\hat{\alpha}_{2} \sin x_{1}+\hat{\alpha}_{7} x_{4}+n_{1} \dot{e}_{1}-\ddot{y}_{m}+\right.$ $\left.+n_{2} e_{2}\right)=\hat{\alpha}_{4}^{-1}\left(x_{1}-y_{m}-\hat{\alpha}_{3} \hat{x}_{2}-\hat{\alpha}_{2} \sin x_{1}+\right.$

$\left.+\hat{\alpha}_{7} x_{4}+n_{1}\left(\hat{x}_{2}-\dot{y}_{m}\right)-\ddot{y}_{m}+n_{2} e_{2}\right)$

where the adaptation laws have the form $\hat{\dot{\alpha}}_{3}=$ $-\gamma_{1} e_{2} \hat{x}_{2}, \hat{\dot{\alpha}}_{2}=-\gamma_{2} e_{2} \sin x_{1}$ with parameters $\hat{\alpha}_{4}=$ $\hat{\alpha}_{2}\left(a_{22} b i\right)^{-1} a_{12} \eta c_{1}, \hat{\alpha}_{7}=\hat{\alpha}_{4} c_{1}^{-1}\left(c_{2}+f_{2}(\eta i)^{-1}\right)$, $n_{2}>0, \gamma_{1}>0, \gamma_{2}>0$.

Substituting (21) for $\dot{V}_{2}$ we get a negative definite function $\dot{V}_{2}=-n_{1} e_{1}^{2}-n_{2} e_{2}^{2}$, so in the $e_{1}=0$ and $e_{2}=0$ system is globally asymptotically stable.
We can obtain the estimation $\hat{J}_{1}$ and then $\hat{f}_{1}$ from the following equations:

$$
\begin{aligned}
& \hat{J}_{1}=\frac{b}{\hat{\alpha}_{2}}+\frac{a_{12}^{2}}{a_{22}}-J_{2}-J_{r}, \\
& \hat{f}_{1}=\frac{\hat{\alpha}_{3}\left(a_{22}\left(\hat{J}_{1}+J_{2}+J_{r}\right)-a_{12}^{2}\right)}{a_{22} i^{-1}} .
\end{aligned}
$$

Since the velocity of the flywheel changes rapidly, we will use the following [Xian et al., 2004] adaptive velocity observers for the pendulum and for the flywheel:

$$
\left\{\begin{array}{l}
\hat{x}_{2}(t)=\hat{\dot{x}}_{1}(t)=p_{x_{2}}(t)+\sigma_{x_{2}} \tilde{x}_{1}(t) \\
\dot{p}_{x_{2}}(t)=\varepsilon_{x_{2}} \operatorname{sign}\left(\hat{x}_{1}(t)\right)+\rho_{x_{2}} \tilde{x}_{1}(t)
\end{array}\right.
$$

$$
\tilde{x}_{1}(t)=x_{1}(t)-\hat{x}_{1}(t)
$$

$$
\left\{\begin{array}{l}
\hat{x}_{4}(t)=\hat{\dot{x}}_{3}(t)=p_{x_{4}}(t)+\sigma_{x_{4}} \tilde{x}_{3}(t) \\
\dot{p}_{x_{4}}(t)=\varepsilon_{x_{4}} \operatorname{sign}\left(\hat{x}_{3}(t)\right)+\rho_{x_{4}} \tilde{x}_{3}(t)
\end{array}\right.
$$

$$
\tilde{x}_{3}(t)=x_{3}(t)-\hat{x}_{3}(t)
$$

where $p(t)_{x_{2}, x_{4}}$ is an auxiliary variable; $\sigma_{x_{2}, x_{4}}, \varepsilon_{x_{2}, x_{4}}, \rho_{x_{2}, x_{4}}$ are constants.

This systems can be used to calculate the angular velocities $\dot{q}_{1}$ and $\dot{q}_{2}$ in (6) instead of directly measuring it by the velocity sensor. For the observer delay compensation the special filters [?] can be used.

\section{Experimental and Simulation Results with Analysis}

In this section we check the algorithms of parameters identification problem.

An experimental device (stand) is shown in Fig.1. We use a $4.5 \mathrm{~W}$ Maxon DC motor; two 14-bit magnetic angular position encoders AS5048A (SPI); an analog amplifier; and a ICP DAS PCI-1202LU data acquisition board. The DC motor is connected to the computer via an isolation converter.

Some parameters of the measuring motor (10) were obtained by Nonlinear Least Squares method separately on the set of free pendulum oscillations and multiharmonic testing signals for the motor: $J_{r}=0.21$. $10^{-5} \mathrm{~kg} \cdot \mathrm{m}^{2}, J_{2}=11.34 \cdot 10^{-5} \mathrm{~kg} \cdot \mathrm{m}^{2}, c_{1}=52.04$. $10^{-4} N \cdot m \cdot V^{-1}, c_{2}=3.697 \cdot 10^{-6} N \cdot \mathrm{m} \cdot \mathrm{rad} / \mathrm{s} \cdot \mathrm{V}^{-2}$, $f_{2}=3.687 \cdot 10^{-5} \mathrm{~N} \cdot \mathrm{m} \cdot \mathrm{s}, \mathrm{f}_{3}=302.02 \cdot 10^{-5} \mathrm{~N} \cdot \mathrm{m}, \eta=$ $0.9, i^{-1}=4.4, d_{1}=5.485 \cdot 10^{-4}, d_{2}=-2.996 \cdot 10^{-4}$. Due to the small parameter $f_{1}$ next we experimentally check the accuracy only of the MOI identification. 
To calibrate the device, we use an additional test body with a known moment of inertia $J_{e}=96.1 \cdot 10^{-5} \mathrm{~kg} \cdot \mathrm{m}^{2}$ and mass $m_{\text {body }}=68.0 \cdot 10^{-3} \mathrm{~kg}, l_{2}=117.0 \cdot 10^{-3} \mathrm{~m}$. This test body can balance the pendulum $(b=0)$ if it is connected to the rod at the opposite distance from point $O$ away from the motor.

In the first case of $J_{e}=0$ which leads to $b=$ $m_{\text {body }} g l_{2}=78 \cdot 10^{-3} \mathrm{~kg} \cdot \mathrm{m}^{3} \cdot \mathrm{s}^{-2}$, using the NLS, the estimated parameter $\hat{J}_{1}=272.6 \cdot 10^{-5} \mathrm{~kg} \cdot \mathrm{m}^{2}$ and using the proposed method $\hat{J}_{1}=302.2 \cdot 10^{-5} \mathrm{~kg} \cdot \mathrm{m}^{2}$. Here we use reference trajectory $y_{m 1}=0.8 \sin (8.5 t)$.

Next we add the test body at the point $C_{2}$, so the parameter $b=2 m_{\text {body }} g l_{2}$ can be easily recalculated, $a_{11}^{\prime}=J_{1}+J_{2}+J_{r}+J_{e}$. Parameter obtained using NLS $\hat{a}_{11}^{\prime}=381.0 \cdot 10^{-5} \mathrm{~kg} \cdot \mathrm{m}^{2}$ and using the proposed method $\hat{a}_{11}^{\prime}=405.1 \cdot 10^{-5} \mathrm{~kg} \cdot \mathrm{m}^{2}$. For this case we use reference trajectory $y_{m 2}=0.8 \sin (6 t)$.

So, we get the estimation of parameter $\hat{J}_{e}=\hat{a}_{11}^{\prime}-$ $\hat{J}_{1}-J_{2}-J_{r}$ using the NLS $\hat{J}_{e}=118.4 \mathrm{~kg} \cdot \mathrm{m}^{2}$ and by the proposed method $\hat{J}_{e}=102.9 \mathrm{~kg} \cdot \mathrm{m}^{2}$. One can see that the experimental identification result is close to the known value with the errors $12 \%$ and $7 \%$, respectively. This results are proven with the balanced pendulum and slow oscillations with the reference trajectory $y_{m 3}=$ $0.8 \sin (2 t)$.

The position tracking errors of the system with the control algorithm (20), system state vector and parameter $\tilde{k}$ are shown in Fig. 2-6. Suppose that the control signal is bounded $\left|u_{0}\right| \leq 10 \mathrm{~V}$, parameters $\delta_{0}=0.08, \sigma_{0}=0.1, \lambda_{0}=2, \overline{\tilde{k}}(0)=0$. For the postprocessing the measured angles in the proposed method passed through a observer in the direct and reverse time for the delay compensation; the control signal passed through a filter with transfer function $W_{f}(p)=(0.0125 p+1)^{-1}$ which is also used in (18) for the smoothing error $e$ in the adaptation.

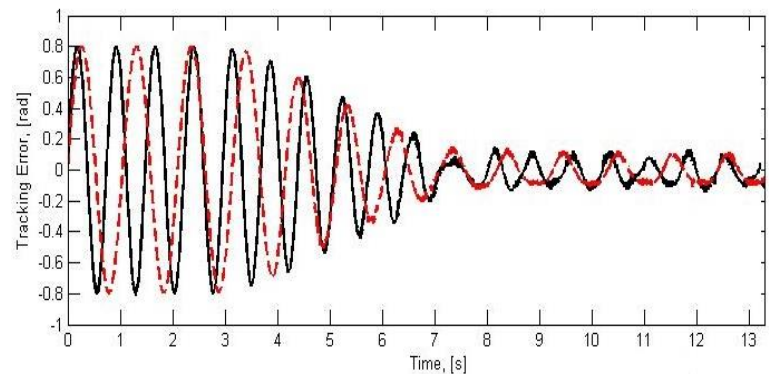

Figure 2. Tracking errors $e$ with (dashed) and without (solid) body (no filtering)

In the proposed method we use series of MOI calculations on a symmetric angular intervals at the time interval from 15 to 22 seconds, where the tuning parameter is no longer changing.

Lets compare the proposed and alternative methods

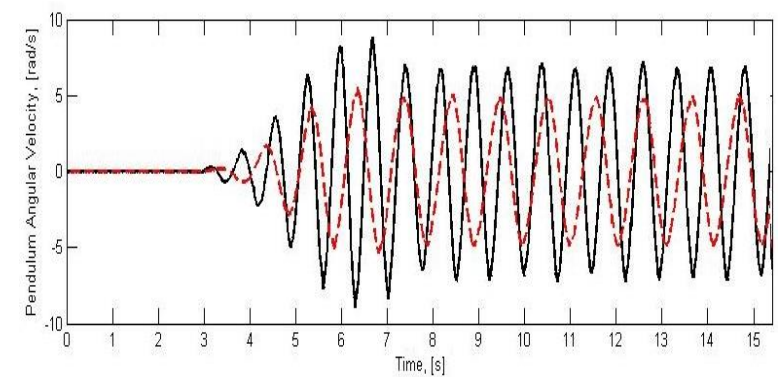

Figure 3. Pendulum angular velocity $\dot{q}_{1}$ with (dashed) and without (solid) body

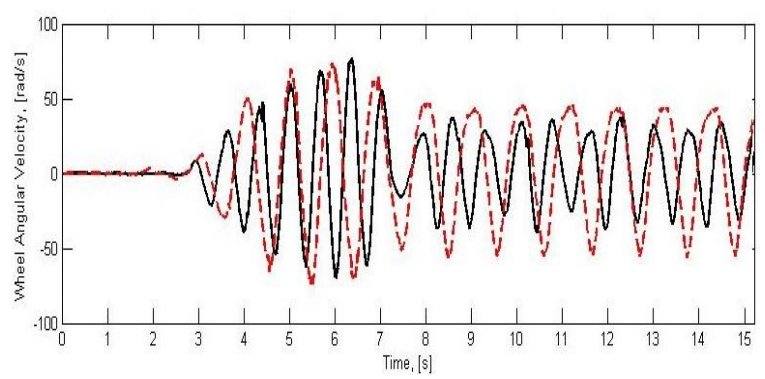

Figure 4. Wheel angular velocity $\dot{q}_{2}$ with (dashed) and without (solid) body

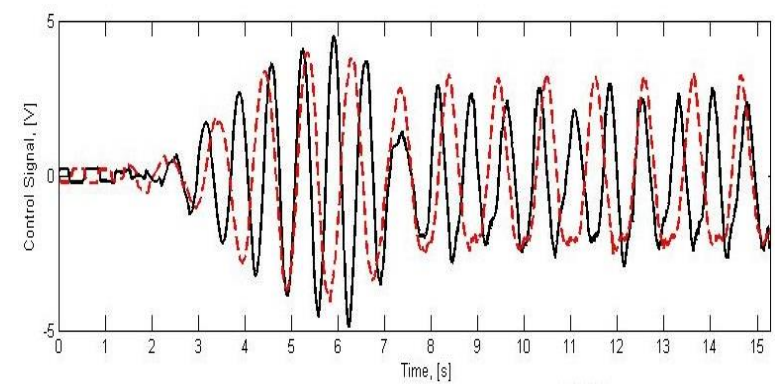

Figure 5. Control signal $u_{0}$ with (dashed) and without (solid) body

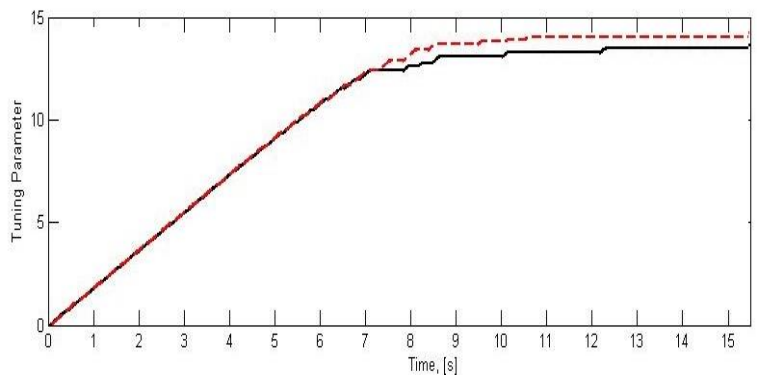

Figure 6. Tuning parameter $\tilde{k}$ complete with filter $W_{f}(p)$

for the MOI ideal identification simulation using the estimated values and friction parameter $f_{1}=26 \cdot 10^{-5} \mathrm{~N}$. $m \cdot s$.

The results of the simulation of the control system 
(20) with the parameters $\delta_{0}=0.005, \sigma_{0}=0.01, \lambda_{0}=$ $150, \tilde{k}(0)=0$ are shown in the Fig. 7 where the angle of the controlled pendulum $q_{1}$ is shown in green, the pendulum reference trajectory is shown in blue, a tracking error of $e$ of the control system is shown in red and $0.005 \tilde{k}$ in brown. Using the calculation formula for the time moments $t_{10}=9.023 \mathrm{~s}, t_{11}=9.413 \mathrm{~s}$, $t_{20}=9.433 \mathrm{~s}, t_{21}=9.823 \mathrm{~s}$ we can get the MOI value $\hat{J}_{1}$ with an average identification error $1,4 \%$.

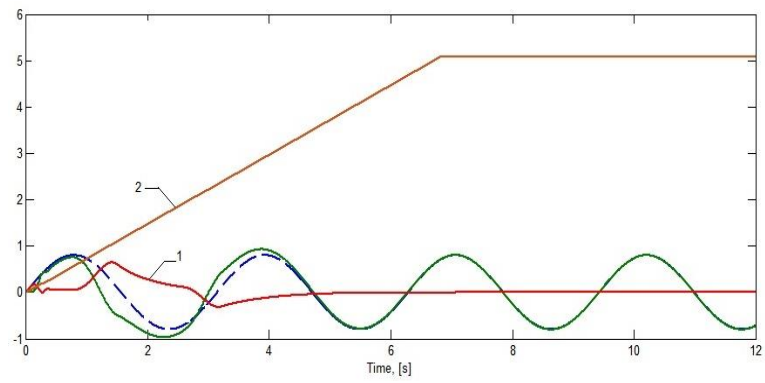

Figure 7. Pendulum angle $q_{1}$ of the control system (solid), program trajectory (dashed), tracking error (1) $e$ and tunable parameter (2).

For the comparison, we simulate a controller containing an observer with tunable parameters (21), (23). The numeric parameter values of the control system are: $\gamma_{2}=100, \gamma_{1}=n_{1}=n_{2}=10$ and of the observers $\sigma_{x_{2}}=\sigma_{x_{4}}=10000, \rho_{x_{2}}=\rho_{x_{4}}=1$, $\varepsilon_{x_{2}}=\varepsilon_{x_{4}}=1$. The initial values of the tunable parameters are $\hat{\alpha}_{2}(0)=1, \hat{\alpha}_{3}(0)=1$. The tunable parameters are limited to positive values $\left[10^{-6} \infty\right]$. The simulation results are shown in Fig. 8. The estimate

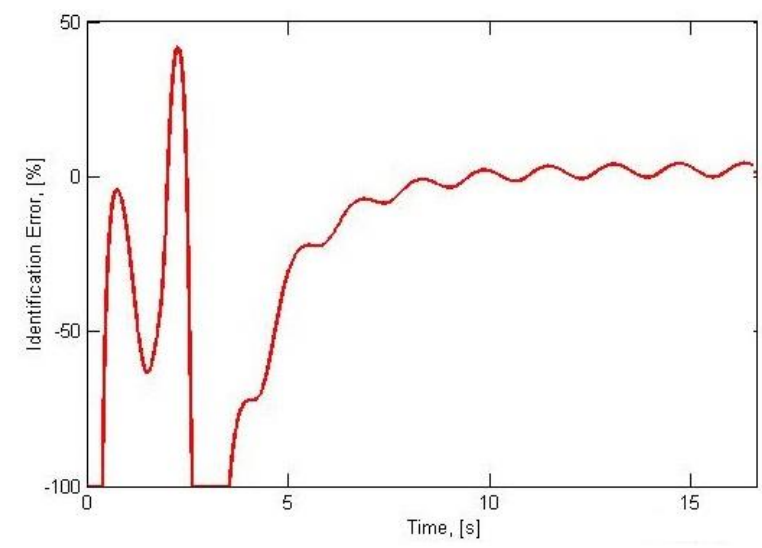

Figure 8. Identification error $J_{1}-\hat{J}_{1}$

of the parameter $\hat{J}_{1}$ converges to the $5 \%$ identification error in $t=8 \mathrm{~s}$. The advantage of this method is that the identification occurs full-online. But controller and observers parameters can be difficult to configure and, due to the possible noise, parameters can be identified incorrectly.

Analysing the experimental and simulation results we conclude that the proposed method with consecutive compensator has several advantages: it does not require measurement of acceleration, cumbersome calculations and linearization; only one two-step symmetric interval is needed to identify the inertia and friction parameters; the dissipative function of any order can be separated from the MOIs; the identification accuracy can be set and estimated by analyzing the tracking error value; the program trajectory can have any convenient symmetric shape with unknown derrivatives; proposed method has a fast-time convergence by selecting the right initial values of tuning parameters, can be easily configured with only two controller parameters, has a stable and small amplitude of the control signal, has high accuracy and can provides greater identification performance than the known method. The method can be also used as a discrete-online identification, where the proposed formula is used for each oscillation.

In the future, we plan to develop multidirectional measuring devices and optimize their control structure [Birk and Dudarenko, 2016, Birk and Dudarenko, 2012].

\section{Conclusion}

This paper presents a new adaptive method for identification of the axial moment of inertia. The developed method is applied to a nonlinear mathematical model of an pendulum system with two degrees of freedom. The method allows to exclude the negative influence of dissipative factors on the accuracy of identification. The proposed method also opens up the possibility of using a convenient and easy-to-install measuring inertial drive for the rapid identification of a wide range of objects. Comparative computer modeling shows that the proposed identification method has higher accuracy and provides greater identification performance. The experimental results are given for illustration.

\section{Acknowledgements}

This work was supported by the Russian Foundation for Basic Research, projects 16-08-00997 and 17-0100672, the state financial support of the leading universities, pr. No. 074-U01, the Ministry of Education and Science pr. No. 14.578.21.0203.

\section{References}

Almeida, R., Urgueira, A., and Maia, N. (2007). Identification of rigid body properties from vibration measurements. Journal of sound and vibration, 299(4), pp. 884-899.

Almeida, R., Urgueira, A., and Maia, N. (2008). Evaluation of the performance of three different meth- 
ods used in the identification of rigid body properties Shock and Vibration, 15(3-4), pp. 467-479.

Alyshev, A., Dudarenko, N., Melnikov, V., and Melnikov, G. (2018). The use of Lyapunov differential inequalities for estimating the transients of mechanical systems. AIP Conf. Proc., 1959:080007.

Alyshev, A. S., Dudarenko, N. A., Melnikov, G. I., and Melnikov, V. G. (2015). Parametric identification of inertial parameters. Applied Mathematical Sciences, 9(136), pp. 6757-6765.

Andrievskiy, B. R. (2004). Stabilization of inverted reaction wheel pendulum. Control in physical and technical systems, Fradkov, A.L., Ed., St. Petersburg: Nauka, pp.52-71.

Andrievskiy, B. R. (2011). Global stabilization of the unstable reaction-wheel pendulum. Automation and Remote Control, 72(9), pp. 1981-1993.

Andrievskiy, B. R. and Boikov, V. I. (2017). Experimental study of multiresonance mechatronic vibrational laboratory set-up. Cybernetics and Physics, 6(1), pp. 5-12.

Barreto, J. and Muñoz, L. E. (2010). Inertia parameter identification using a stewart platform. 6th German Conf. on Robotics (ROBOTIK), pp. 1-8.

Benaskeur, A. and Desbiens, A. (1998). Application of adaptive backstepping to the stabilization of the inverted pendulum. IEEE Canadian Conf. on Electrical and Computer Eng., 1, pp. 113-116.

Beznos, A. V., Grishin, A. A., Lenskii, A. V., Okhotsimsky, D. E., and Formalskii, A. M. (2003b). A flywheel usebased control for a pendulum with fixed suspension point. J. Comput. Syst. Sci. Int., (43(1)), pp. 2233.

Beznos, A. V., Grishin, A. A., Lenskii, A. V., Okhotsimsky, D. E., and Formalskii, A. M. (2003a). A pendulum controlled by a flywheel. Dokl. Math., (68(2)), pp. 302307.

Birk, W. and Dudarenko, N. (2012). Evaluation of two methods for reconfiguration of multivariable controllers in process control systems. 2012 IEEE Int. Conf. on Control Applications (CCA), pp. 627-633.

Birk, W. and Dudarenko, N. (2016). Reconfiguration of the air control system of a bark boiler. IEEE Transactions on Control Systems Technology, 24(2), pp. 565577.

Block, D. J., Åström, K. J., and Spong, M. W. (2007). The reaction wheel pendulum. Synthesis Lectures on Control and Mechatronics, 1(1), pp. 1-105.

Bobtsov, A. and Pyrkin, A. (2008). Experimental research of consecutive compensator approach on basis of mechatronic systems. 6th EUROMECH Nonlinear Dynamics Conf.

Bobtsov, A. A., Kolyubin, S. A., and Pyrkin, A. A. (2011). Stabilization of the Schmid pendulum on the movable platform with real-time controller adjustment and adaptive friction compensation. IFAC Proc. Volumes, 44(1), pp. 4137-4142.

Chiu, C.-H., Peng, Y.-F., and Lin, Y.-W. (2011). Robust intelligent backstepping tracking control for wheeled inverted pendulum. Soft Computing, 15(10), pp. 2029-2040.

Dudarenko, N., Melnikov, V., and Melnikov, G. (2014). A method for inertia tensors and centres of masses identification on symmetric precessions. 6th Int. Congress on Ultra Modern Telecommunications and Control Systems and Workshops (ICUMT), pp. 596601.

Ebrahim, A. and Murphy, G. V. (2005). Adaptive backstepping controller design of an inverted pendulum. Proc. of SSST'05., pp. 172-174.

Fradkov, A. and Andrievsky, B. (2004). Singular perturbations of systems controlled by energy-speedgradient method. Proc. of the IEEE Conf. on Decision and Control, 4, pp. 3441-3446.

Fradkov, A. and Andrievsky, B. (2006). Singular perturbation analysis of energy control systems. JVC/Journal of Vibration and Control, 12(4), pp. 331-353.

Fradkov, A. L., Andrievsky, B., and Boykov, K. B. (2012). Multipendulum mechatronic setup: Design and experiments. Mechatronics, 22(1), pp. 76-82.

Garćia-Alarćon, O., Puga-Guzman, S., and MorenoValenzuela, J. (2012). On parameter identification of the furuta pendulum. Procedia Engineering, 35, pp. 77-84.

Gobbi, M., Mastinu, G., and Previati, G. (2011). A method for measuring the inertia properties of rigid bodies. Mechanical Systems and Signal Processing, 25(1), pp. 305-318.

Hou, Z.-C., Lu, Y.-n., Lao, Y.-X., and Liu, D. (2009). A new trifilar pendulum approach to identify all inertia parameters of a rigid body or assembly. Mechanism and Machine Theory, 44(6), pp. 1270-1280.

Ivanov, S. and Melnikov, V. (2015). Mathematical modeling vibration protection system for the motor of the boat. Applied Mathematical Sciences, 9(117120), pp. 5951-5960.

Krstic, M., Kanellakopoulos, I., and Kokotovic, P. V. (1995). Nonlinear and adaptive control design. Wiley.

Malekjafarian, A., Ashory, M., Khatibi, M., and SaberLatibari, M. (2016). Rigid body stiffness matrix for identification of inertia properties from output-only data. European Journal of Mechanics-A/Solids, 59, pp. 85-94.

Melnikov, V. (2010). Chebyshev economization in transformations of nonlinear systems with polynomial structure. Int. Conf. on Systems - Proc., 1, pp. 301-303.

Melnikov, V. (2012). Inertia tensors and centres of masses identification at semiprogram precession motions. Proc. IEEE Int. Conf. on Control Applications, pp. 494-497.

Ovchinnikov, V., Nikolaev, M., and Litvinov, V. (2017). Algorithm for compensation of residual imbalance of 
a flexible rotor on active magnetic bearings. Cybernetics and Physics, 6(2), pp. 76-79.

Pandit, S., Hu, Z.-Q., and Yao, Y.-X. (1992). Experimental technique for accurate determination of rigid body characteristics. Proc. of the Int. modal analysis Conf., pp. 307-307.

Spong, M. W., Corke, P., and Lozano, R. (2001). Nonlinear control of the reaction wheel pendulum. Automatica, 37(11), pp. 1845-1851.

Tikhonov, A. (2000). Resonance phenomena in oscillations of a gravity-oriented rigid body. part 4: Multi- frequency resonances. Vestnik Sankt-Peterburgskogo Universiteta. Ser 1. Matematika Mekhanika Astronomiya, (1), pp. 131-137.

Tikhonov, A. and Tkhai, V. (2016). Symmetrical oscillations of charged gyrostat in weakly elliptical orbit with small inclination. Nonlinear Dynamics, 85(3), pp. 1919-1927.

Xian, P., Queiroz, M. S., Dawson, D. M., and Mclntyre, M. L. (2004). A discontinuous output controller and velocity observer for nonlinear mechanical systems. Automatica, 40, pp. 695-700. 